Old Dominion University

ODU Digital Commons

Mechanical \& Aerospace Engineering Faculty

Publications

Mechanical \& Aerospace Engineering

2018

Electrical Response of Thermoelectric Generator to Geometry

Variation Under Transient Thermal Boundary Condition

Elias Yazdanshenas

Alireza Rezania

Meysam Karami Rad

Lasse Rosendahl

Follow this and additional works at: https://digitalcommons.odu.edu/mae_fac_pubs

Part of the Electro-Mechanical Systems Commons, Energy Systems Commons, and the Heat Transfer, Combustion Commons 
Electrical response of thermoelectric generator to geometry variation under transient thermal boundary condition

Elias Yazdanshenas, Alireza Rezania, Meysam Karami Rad, and Lasse Rosendahl

Citation: Journal of Renewable and Sustainable Energy 10, 064705 (2018); doi: 10.1063/1.5040166

View online: https://doi.org/10.1063/1.5040166

View Table of Contents: http://aip.scitation.org/toc/rse/10/6

Published by the American Institute of Physics

\section{Don't let your writing} keep you from getting published! 


\title{
Electrical response of thermoelectric generator to geometry variation under transient thermal boundary condition
}

\author{
Elias Yazdanshenas, ${ }^{1}$ Alireza Rezania, ${ }^{2, a)}$ Meysam Karami Rad, ${ }^{2,3}$ and \\ Lasse Rosendahl ${ }^{2}$ \\ ${ }^{1}$ Department of Mechanical \& Aerospace Engineering, Old Dominion University, \\ Norfolk, Virginia 23529, USA \\ ${ }^{2}$ Department of Energy Technology, Aalborg University, Pontoppidanstrade 111, \\ Aalborg DK-9220, Denmark \\ ${ }^{3}$ Laboratory of Organic Electronics, Department of Science and Technology, \\ Linköping University, 60174 Norrköping, Sweden
}

(Received 15 May 2018; accepted 28 November 2018; published online 18 December 2018)

\begin{abstract}
A three-dimensional numerical model is applied in this study to illustrate the electrical response of a thermoelectric generator (TEG) during transient heat flux at the hot side. In this work, various types of thermal boundary conditions are considered to evaluate the performance of the TEG. Thus, a TEG under pulsed heat flux is studied numerically, and the numerical model is verified by experimental results. With the consideration of a defined reference geometry, different heat flux frequencies are applied in order to evaluate the corresponding electrical output by the TEG. In addition, variation of the module performance for various TEG leg lengths and its cross-sectional area are studied over a wide range of heat fluxes. The results indicate that the open circuit voltage in the experiment is in a good agreement with the open circuit voltage in the simulation results. The results show that the range of power oscillation reduces at higher frequency of the applied heat flux. Furthermore, the variability of the output power increases as the thermoelectric element length increases and the area of the element reduces. Published by AIP Publishing. https://doi.org/10.1063/1.5040166
\end{abstract}

\section{INTRODUCTION}

A thermoelectric generator (TEG) is a device that works on the principle of the Seebeck effect, enabling it to convert thermal energy directly into electricity. Many researchers have considered a wide range of applications for thermoelectric devices. Wang et al. ${ }^{1}$ and Qing et $a .^{2}{ }^{2}$ investigated a TEG which was a full-fledged wearable for the human body. Amatya and $\mathrm{Ram}^{3}$ linked solar parabolic concentrators to a Hi-Z thermoelectric to produce electrical power. Samson $\mathrm{et} \mathrm{al}^{4}{ }^{4}$ studied TEG for energy harvesting in an aircraft to supply energy for the wireless sensor node. Goudarzi et al. ${ }^{5}$ used the TEG for harvesting waste heat from domestic wood stoves. In automotive heat recovery applications, Bass et al. ${ }^{6}$ used 72 high impedance (Hi-Z) $13 \mathrm{~W}$ bismuth-telluride thermoelectrics for truck engines in order to generate a power of $1 \mathrm{~kW}$.

Components of typical TEG modules contain n-type and p-type semiconductor legs connected in series electrically and sandwiched between two layers of ceramic substrates. By applying thermal energy on one side and connecting the other side to a heat sink, the temperature difference created across the module causes charge carriers to migrate from the hightemperature side to the low temperature side; thus, the different amount of charges on the cold and hot sides generates an internal electrical field. ${ }^{7}$

In most studies in the field of thermoelectrics, heat load and imposed heat flux on the hot side of the TEG have been considered the steady state, ${ }^{8-10}$ while the heat source has transient

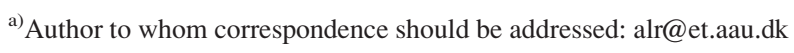


behavior in many practical applications. Thus, the amount of demand for various applications has been limited. To expand the application of TEGs, transient heat flux has been considered ${ }^{11-13}$ also with phase change materials ${ }^{14}$ in heat recovery from industries such as car brake manufacturing and pulsed laser in materials processing. ${ }^{15}$ Yamashita et al. ${ }^{16}$ showed that the efficiency of TEGs can be enhanced by the application of periodic temperature. Therefore, input temperature on the hot side is one of the parameters with a high impact on the TEG performance.

Although the cold side temperature is affected by the hot side temperature, the performance of TEGs enhances as the applied heat flux and, thus, the temperature on the hot side increase. Nevertheless, if the temperature difference between the two sides enhances, the conversion performances can be improved. Therefore, in the TEG systems, not only the optimal design of the heat sink and heat source is important, but also optimization of the TEG module is simultaneously critical in order to enhance electrical output of the TEG. ${ }^{17,18}$ One of the key parameters affecting the performance of TEG is the geometry of the thermoelectric elements. Min and Rowe ${ }^{19}$ chose three types of commercial TEGs with various lengths at a temperature of $120^{\circ} \mathrm{C}$ from the heat sources. By 55\% reduction in the TEG length, they achieved 48\% improvement in electrical power output. Sahin and Yilbas ${ }^{20}$ and Rezania et al. ${ }^{21}$ addressed a shape parameter which relates the output electricity of TEG and geometry of thermoelectric elements. They concluded that the device efficiency can be influenced considerably by changing the shape parameter of the TEG leg.

Nevertheless, state-of-the-art in this field lacks the study on the effect of TEG geometry on its electrical response based on the properties of the applied heat pulse. Thus, the width and the depth of the TEG elements can have an impact on the efficiency of the TEG, so that 3D simulation of the TEG is required for a detailed study. In the present work, several types of transient heat fluxes are applied on the TEG, and electrical response and performance of the TEG are studied. Then, a 3D model of the TEG with different thermal boundary conditions is simulated under various types of periodic heat on the hot side of the TEG. Furthermore, the performance of the TEG from simulation and experimental results is compared for the validation study in this work.

\section{COMPUTATIONAL METHOD}

The model for thermoelectric can be expressed by the heat transfer equation ${ }^{22}$

$$
\rho C \frac{\partial T}{\partial t}+\nabla \cdot q^{\prime \prime}=Q^{\prime}
$$

where $\rho, C, T, t, q^{\prime \prime}$, and $Q^{\prime}$ are the density, C heat capacity, temperature, time, heat flux, and density of Joule heating energy, respectively. The continuity of electric current is expressed as

$$
\nabla . J=\frac{\partial \rho_{c}}{\partial t},
$$

where $\rho_{\mathbf{c}}$ denotes the charge density and $\mathbf{J}$ is the electric current flux. The joule heating energy is expressed as follows:

$$
\begin{gathered}
Q^{\prime}=J . E, \\
q^{\prime \prime}=-k \nabla T+P^{\prime} J,
\end{gathered}
$$

where $E$ and $P^{\prime}$ denote the electric field and the Peltier coefficient, respectively, which depend on the reversible Seebeck effect and the irreversible Joule effect

$$
\begin{gathered}
P^{\prime}=S T, \\
J=-\sigma \nabla V-\sigma S \nabla T .
\end{gathered}
$$


TABLE I. Material properties used in the simulation.

\begin{tabular}{lcccccc}
\hline \hline Material & $\begin{array}{c}\text { Electrical } \\
\text { conductivity }(\mathrm{S} / \mathrm{m})\end{array}$ & $\begin{array}{c}\text { Heat capacity } \\
\text { at constant } \\
\text { pressure }\left(\frac{\mathrm{J}}{\mathrm{kg} \cdot \mathrm{K}}\right)\end{array}$ & $\begin{array}{c}\text { Density } \\
\left(\frac{\mathrm{kg}}{\mathrm{m}^{3}}\right)\end{array}$ & $\begin{array}{c}\text { Thermal } \\
\text { conductivity }\left(\frac{\mathrm{W}}{\mathrm{m} \cdot \mathrm{K}}\right)\end{array}$ & $\begin{array}{c}\text { Seebeck } \\
\text { coefficient }\left(\frac{\mathrm{V}}{\mathrm{K}}\right)\end{array}$ & $\begin{array}{c}\text { Relative } \\
\text { conductivity }\end{array}$ \\
\hline Copper & $5.998 \times 10^{7}$ & 385 & 8700 & 400 & & \\
Bismuth telluride-BiTe3 & Sigma $(\mathrm{T})^{\mathrm{a}}$ & 154 & 7700 & $\mathrm{k}(\mathrm{T})^{\mathrm{b}}$ & $p /$ ntype $: \pm \mathrm{S}(\mathrm{T})^{\mathrm{c}}$ & 1 \\
Alumina-AD-995 & & 880 & 3700 & 30 & & \\
\hline \hline
\end{tabular}

${ }^{\mathrm{a}} \operatorname{Sigma}(\mathrm{T})=-4 \times 10^{-15} T^{6}+10^{-11} T^{5}-10^{-8} T^{4}+7 \times 10^{-6} T^{3}-0.002 T^{2}+0.3283 T-19.919$.

${ }^{\mathrm{b}} \mathbf{K}(\mathbf{T})=4 \times 10^{-17} \boldsymbol{T}^{6}-4 \times 10^{-12} \boldsymbol{T}^{5}+10^{-8} \boldsymbol{T}^{4}-9 \times 10^{-6} \boldsymbol{T}^{3}+0.0038 \boldsymbol{T}^{2}-0.8272 \boldsymbol{T}+73.158$.

${ }^{\mathrm{c}} \mathrm{S}(\mathrm{T})= \pm\left(-3 \times 10^{-18} T^{6}+7 \times 10^{-15} T^{5}-7 \times 10^{-12} T^{4}+4 \times 10^{-9} T^{3}-10^{-6} T^{2}+0.0002 T-0.0167\right)$.

By substituting Eqs. (3) and (6) into Eqs. (1) and (2), the thermoelectric equations can be written as follows:

$$
\begin{gathered}
\rho C \frac{\partial \boldsymbol{T}}{\partial t}+\nabla \cdot(-k \nabla T+S T(-\sigma \nabla V-\sigma S \nabla T))=(-\sigma \nabla V-\sigma S \nabla T)(-\nabla V), \\
-\sigma\left(\nabla^{2} V+S \nabla^{2} T\right)=\frac{\partial \rho_{c}}{\partial t} .
\end{gathered}
$$

The material properties of the thermoelectric model in the simulations and the TEG with 97 uni-couples used in the experiments are shown in Table I.

The maximum power generation by the TEG is ${ }^{23}$

$$
\dot{W}_{\max }=\frac{S^{2} \Delta T^{2}}{4 R},
$$

where the $S \Delta T$ term represents the Seebeck voltage and $R$ is the total internal electrical resistance of the TEG

$$
R=\frac{\rho_{e p} L_{p}}{A_{p}}+\frac{\rho_{e n} L_{n}}{A_{n}}
$$

In Eq. (10), $R_{p}, L_{p}, A_{p}, R_{n}, L_{n}$, and $A_{n}$ denote the electrical resistivity in p-type, length of $\mathrm{p}$ type, area of the p-type, electrical resistivity in n-type, length of n-type, and area of the ntype.

\section{BOUNDARY CONDITIONS}

In the present work, a uni-couple TEG is simulated by COMSOL Metaphysics. As shown in Fig. 1, heat transfer and electric current from the coupled mechanism predicts the device performance. The temperature on the higher surface of the top ceramic, in contact with the heat source, is identified by the variation of the applied heat flux. A fixed value of the heat transfer coefficient equal to $50 \mathrm{KW} / \mathrm{m}^{2} . \mathrm{K}$ is applied to the cold side of the bottom ceramic substrate. The initial temperature of the components and the ambient temperature are taken to be $300 \mathrm{~K}$. In this work, the space among the thermoelements in the TEG used for the experiments is filled by the non-conductive insulation material; therefore, heat loss from the component wall exposed to the other elements and surrounding is taken to be zero, $-n . q=0$. Table II shows the dimensions of the TEG components used in this study.

\section{EXPERIMENTAL SETUP AND VALIDATION}

An experimental setup [see Fig. 2(a)] is used to study the transient behavior of the TEG. In order to reduce the heat losses from the side of the TEG, the experiments are carried out in a 


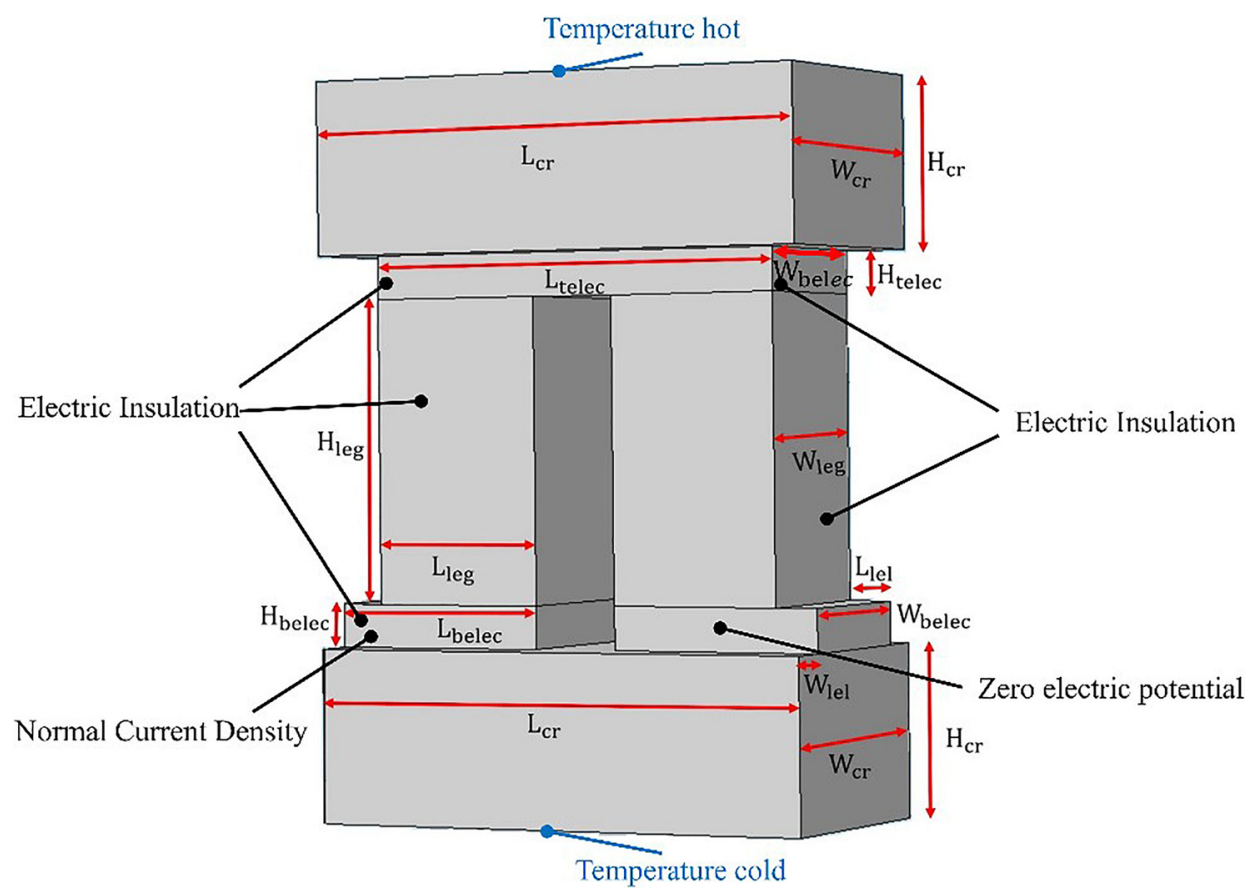

FIG. 1. Boundary condition of the simulated uni-couple and the TEG used in the experiments with the same dimensions as mentioned in Table II.

vacuum chamber that provides thermal insulation for the system from the ambient. An ULTRAMIC 600 heater supplied by Keysight B2900-smu is used to apply the heat flux to the TEG and to keep the hot side of the TEG at the desired temperature. Due to high power intensity and low thermal mass of the heater, it is possible to provide a fast thermal load with high temperature differences on the hot side of the TEG. The temperatures are sensed by T-type thermocouples with a diameter of $50 \mu \mathrm{m}$ and a nominal time response of $20 \mathrm{~ms}$. The Keysight 34972 data logger is used to $\log$ data every $30 \mathrm{~ms}$. The experimental setup shown in Fig. 2(b) contains the heater, cooler, and measurement system. Silicon thermal paste with a thermal conductivity of 3.1 $\mathrm{W} / \mathrm{m} \mathrm{K}$ is used to guarantee the proper thermal contact between heat transfer layers.

In the experiments, the change in the temperature on the cold and hot sides with respect to time is shown in Fig. 3. The hot side temperature of the TEG starts to rise from $23{ }^{\circ} \mathrm{C}$ and continues with a periodic shape to its maximum temperature at $80{ }^{\circ} \mathrm{C}$; then, at time equal to $125 \mathrm{~s}$, the temperature decreases gradually to reach $23{ }^{\circ} \mathrm{C}$. A large heat sink, in comparison with the size of the TEG, is used in the experiments; therefore, the cold side temperature remains almost constant, while the hot side temperature changes. By applying the temperatures on the hot and cold sides of the TEG in the simulation, the results show that the open circuit voltage behavior follows electrical potential in the experiments. The open circuit voltage at the beginning of the test is $0 \mathrm{~V}$, while its trend follows temperature variations on the hot side. The error according to the root-mean-square deviation (RMSD) method $^{24}$ in experimental and simulation results is 5.44 .

TABLE II. Dimensions of the TEG components.

\begin{tabular}{lccccccc}
\hline \hline Parameter & $W_{c r}$ & $L_{c r}$ & $H_{c r}$ & $W_{\text {leg }}$ & $L_{\text {leg }}$ & $H_{\text {leg }}$ & $W_{\text {telec }}$ \\
\hline Size $(\mathrm{mm})$ & 0.6 & 1.2 & 0.1 & 0.4 & 0.4 & 0.7 & 0.4 \\
Parameter & $L_{\text {telec }}$ & $H_{\text {telec }}$ & $W_{\text {belec }}$ & $L_{\text {belec }}$ & $H_{\text {belec }}$ & $L_{\text {lel }}$ & $W_{\text {lel }}$ \\
\hline Size $(\mathrm{mm})$ & 1 & 0.05 & 0.4 & 0.5 & 0.1 & 0.1 & 0.1 \\
\hline \hline
\end{tabular}




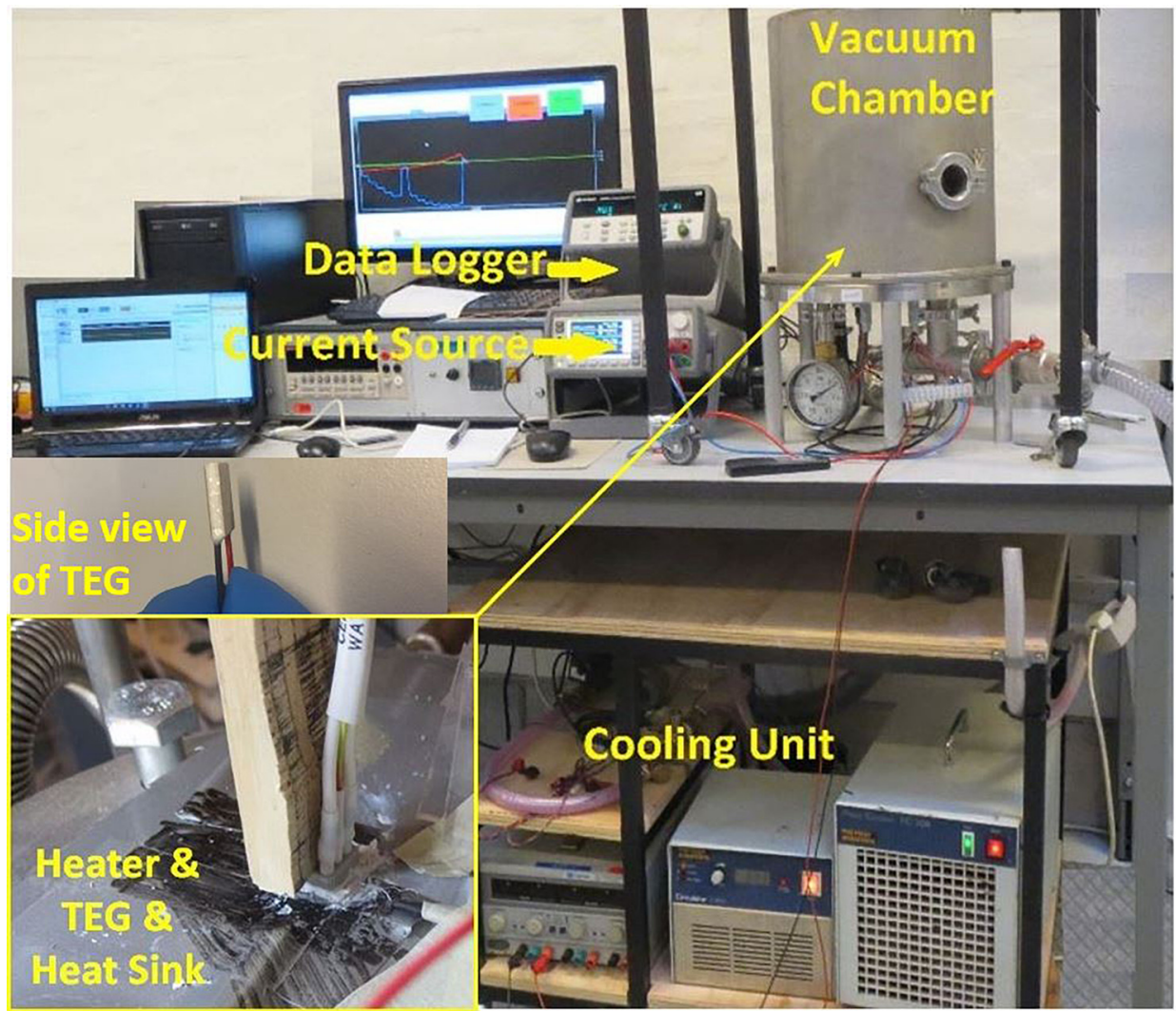

(a)

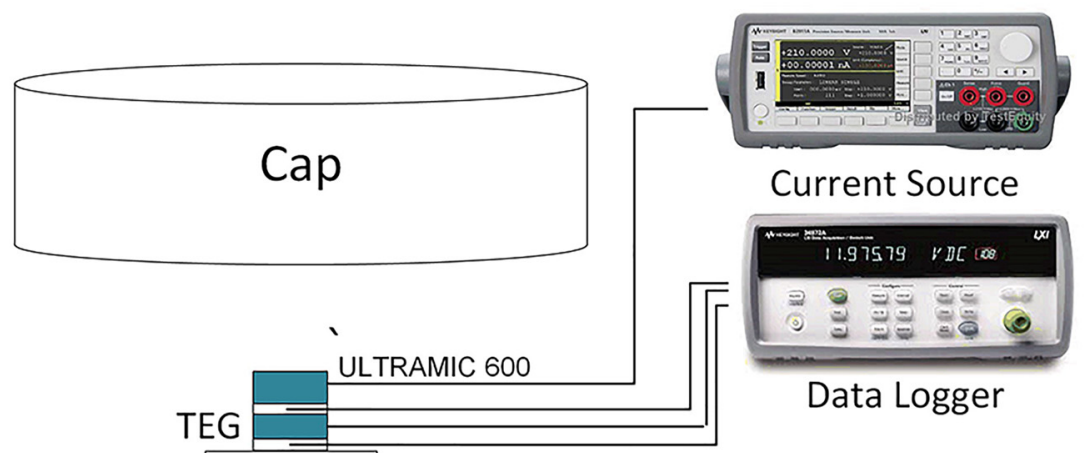

(b)

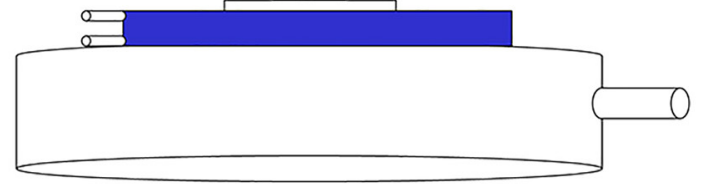

FIG. 2. (a) Experimental setup and (b) schematic of the test setup with the configuration of the heater, TEG, and heat sink.

\section{RESULTS AND DISCUSSION}

To examine the effect of the thermal load on the output voltage of the TEG, two sample times are chosen from the reference geometry with a frequency of $0.25 \mathrm{~Hz}$, while a heat flux of $150 \mathrm{KW} / \mathrm{m}^{2}$ is applied on the TEG hot side. Figure 4 shows the temperature and voltage distribution in a studied uni-couple. As the temperature on the hot side drops at $\mathrm{t}=28.3 \mathrm{~s}$, the open circuit voltage follows the temperature variation and decreases. The highest temperature at the time of $30 \mathrm{~s}$ generates the highest open circuit voltage in the uni-couple. 


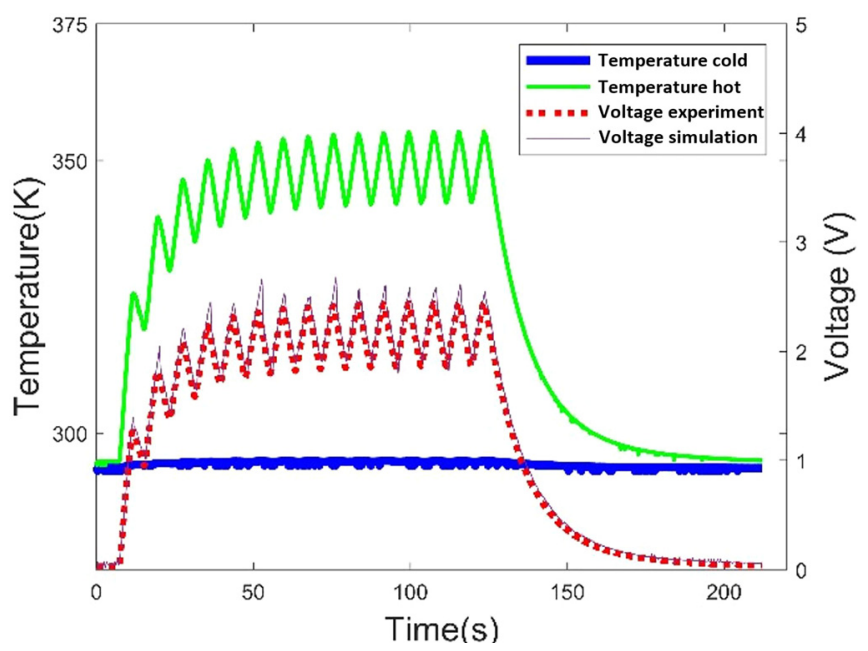

FIG. 3. Comparison between simulation and experimental results of open circuit voltage in TEG with 97 uni-couples under the transient thermal boundary condition.

Since external surfaces of the thermoelectric legs in the simulations are assumed to be insulated from the surrounding, the internal heat transfer, such as natural convection and radiative heat transfers, among the thermoelectric legs is zero. Therefore, as shown in Fig. 5, the temperature variation along the thermoelectric leg is linear. The temperature at the cold side of the TEG is constant over time due to the high heat transfer coefficient imposed at the cold side of the TEG, while the hot side temperature changes over time as the applied heat flux fluctuates. The thermal conductivity of the ceramic substrates and the interconnectors is large compared to the thermal conductivity of the semiconductor legs, and therefore, the temperature from the top

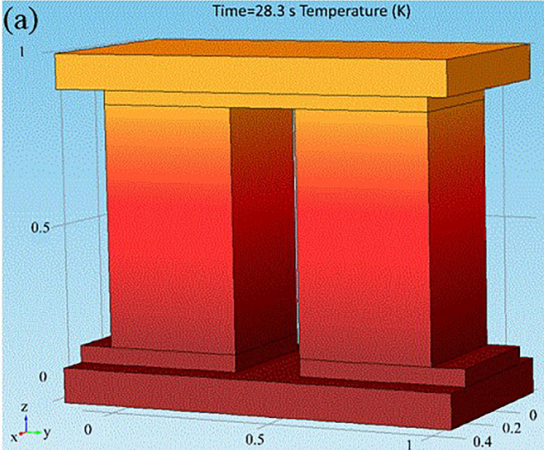

(c)

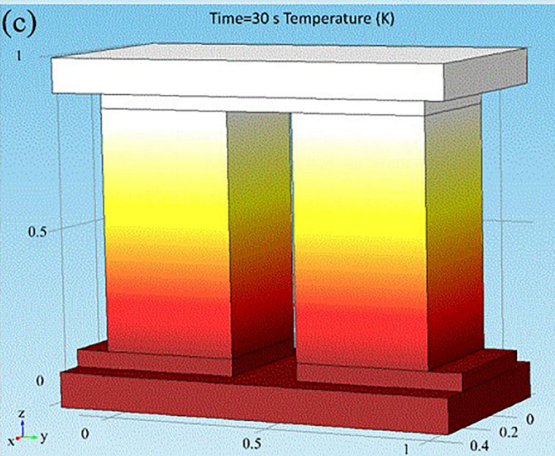

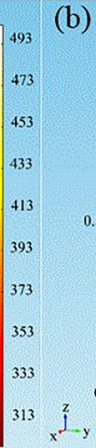
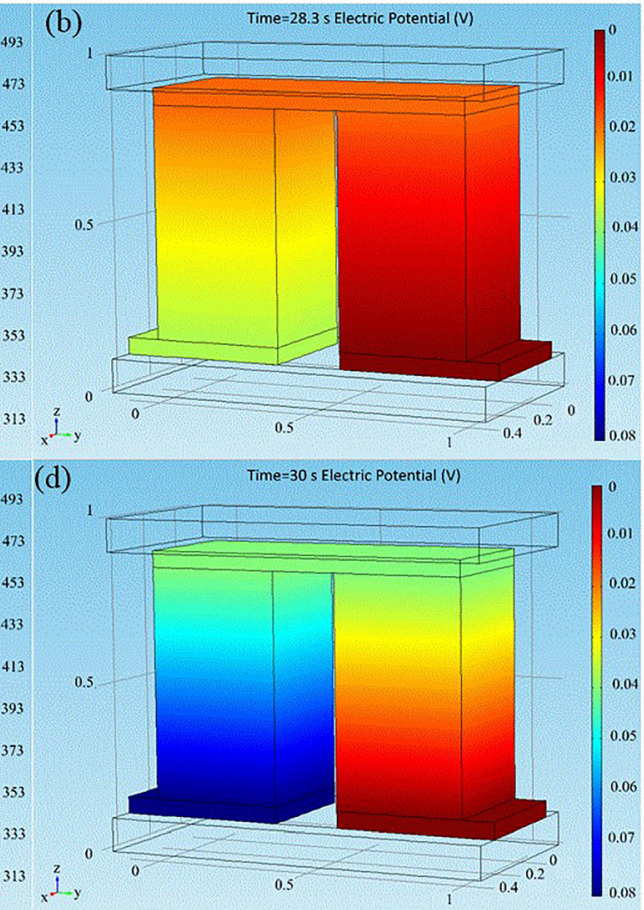

FIG. 4. The temperature and electrical potential on thermoelectric elements at the frequency of $0.25 \mathrm{~Hz}$ : (a) the temperature contour at $28.3 \mathrm{~s}$, (b) electrical potential contour at $28.3 \mathrm{~s}$, (c) the temperature contour at $30 \mathrm{~s}$, and (d) electrical potential contour at $30 \mathrm{~s}$. 


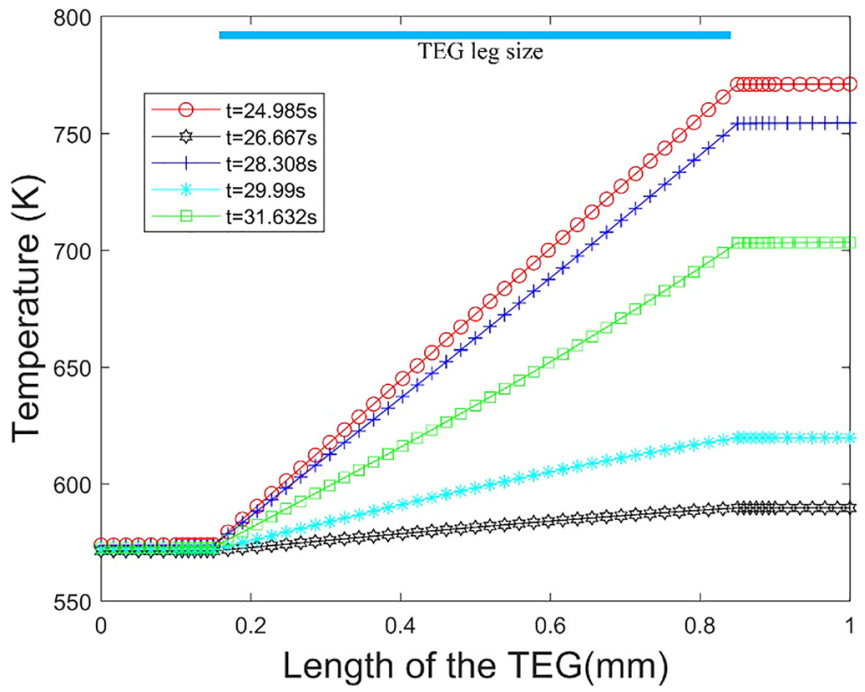

FIG. 5. Temperature profile along the centerline of the thermoelectric elements at $t=24.985 \mathrm{~s}, \mathrm{t}=26.667 \mathrm{~s}, \mathrm{t}=28.308 \mathrm{~s}$, $\mathrm{t}=29.99 \mathrm{~s}$, and $\mathrm{t}=31.632 \mathrm{~s}$, heat flux $=150 \mathrm{KW} / \mathrm{m}^{2}$, and frequency $=0.25 \mathrm{~Hz}$.
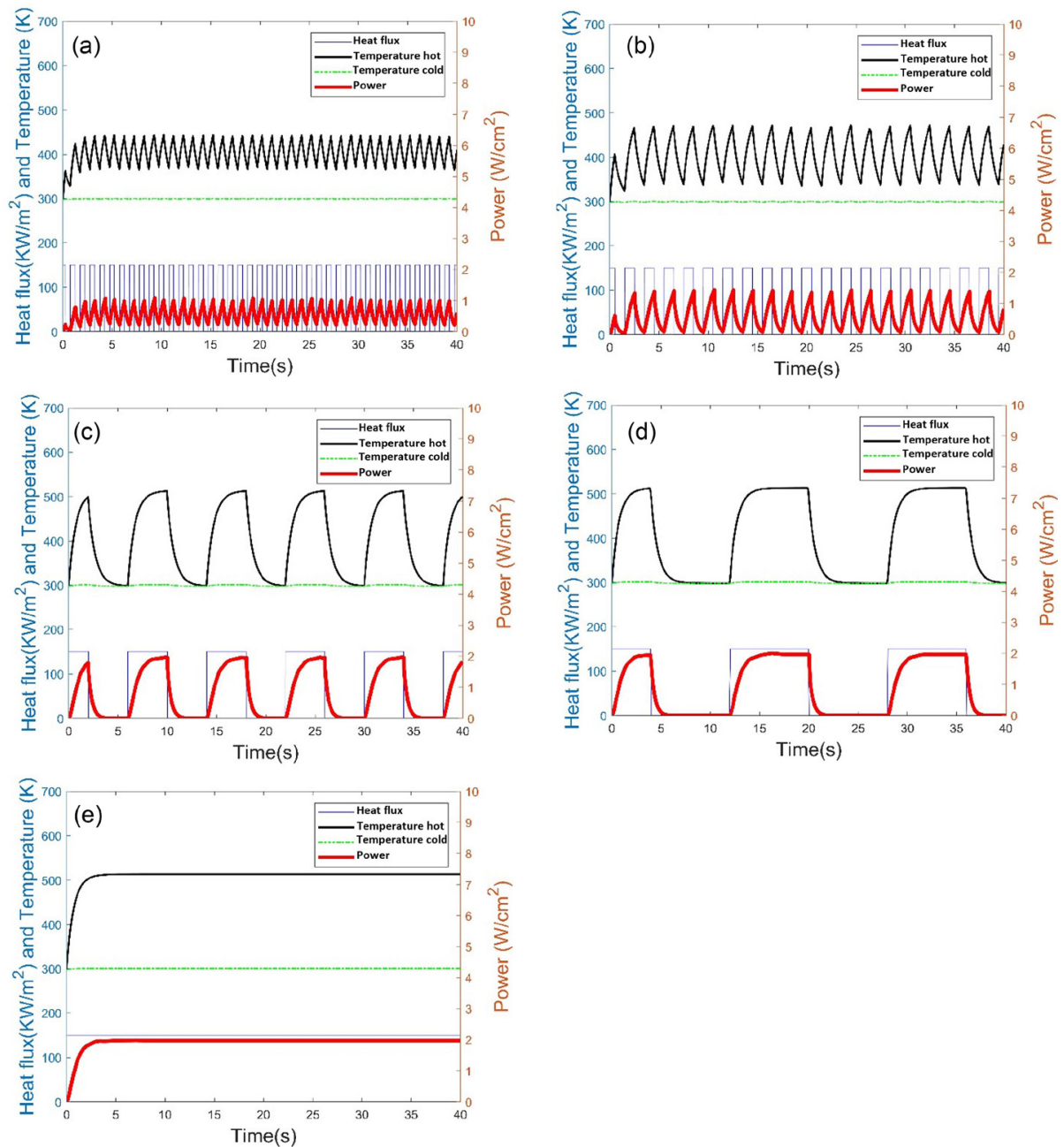

FIG. 6. Variation of open circuit power and temperature of the cold and hot sides of the reference geometry at different heat flux frequencies, (a) $\mathrm{f}=1 \mathrm{~Hz}$, (b) $\mathrm{f}=0.5 \mathrm{~Hz}$, (c) $\mathrm{f}=0.125 \mathrm{~Hz}$, (d) $\mathrm{f}=0.0625 \mathrm{~Hz}$, and (e) constant heat flux. 
and bottom surfaces of the TEG to the hot and cold sides of the legs, respectively, remains almost constant in compression with the temperature variation along the legs.

Sensitivity of thermo-electrical response of the TEG to the pulse frequency is considered for the reference geometry. Figure 6 shows the variation of the temperature and power for 4 pulse frequencies. The results of compression among the studied frequencies indicate that lower frequency creates higher fluctuation in temperature and power generation. The electrons migrate more as the temperature difference of the leg is higher. Additionally, the power variation is linked to the temperature of the hot and cold sides according to Eq. (6). Although the range and average amount of applied heat flux in this study are the same for different frequencies, the range of temperature and power variations is wider for lower frequency. This variation can depend on the material properties such as thermal diffusion [see Eq. (7)].

The effect of different element lengths $(0.1 \mathrm{~mm}, 0.4 \mathrm{~mm}, 1 \mathrm{~mm}$, and $1.3 \mathrm{~mm})$ is furthermore considered in order to make a comparison among the performance of various TEG designs as shown in Fig. 7. The boundary conditions in these simulations are the same as the reference case. By applying a pulse frequency of $0.25 \mathrm{~Hz}$ and the reference heat flux on the hot side of the TEG, the highest power is gained for the highest length, which has the highest thermal resistance among the considered lengths. The Seebeck voltage depends on material properties and dimension of the semiconductor and enhances as the length of the leg and, consequently, the temperature difference between hot and cold sides increase. ${ }^{25}$ The Seebeck voltage is also a geometry property and decreases as the leg length reduces. Since the power generation, moreover, follows the temperature difference, it reaches zero when the hot side temperature of the TEG is given sufficient time to reach the cold side temperature. This form of power generation profile is seen for leg lengths shorter than $0.4 \mathrm{~mm}$ at $\mathrm{f}=0.25 \mathrm{~Hz}$.

For the reference geometry and under the same thermal boundary conditions, the effect of different leg areas is also considered. As shown in Fig. 7, by considering parameters of the length of the TEG, when the area of TEG increases under fixed heat flux, the hot side temperature drops to balance the energy flow across the legs, so that the voltage generation reduces. The results
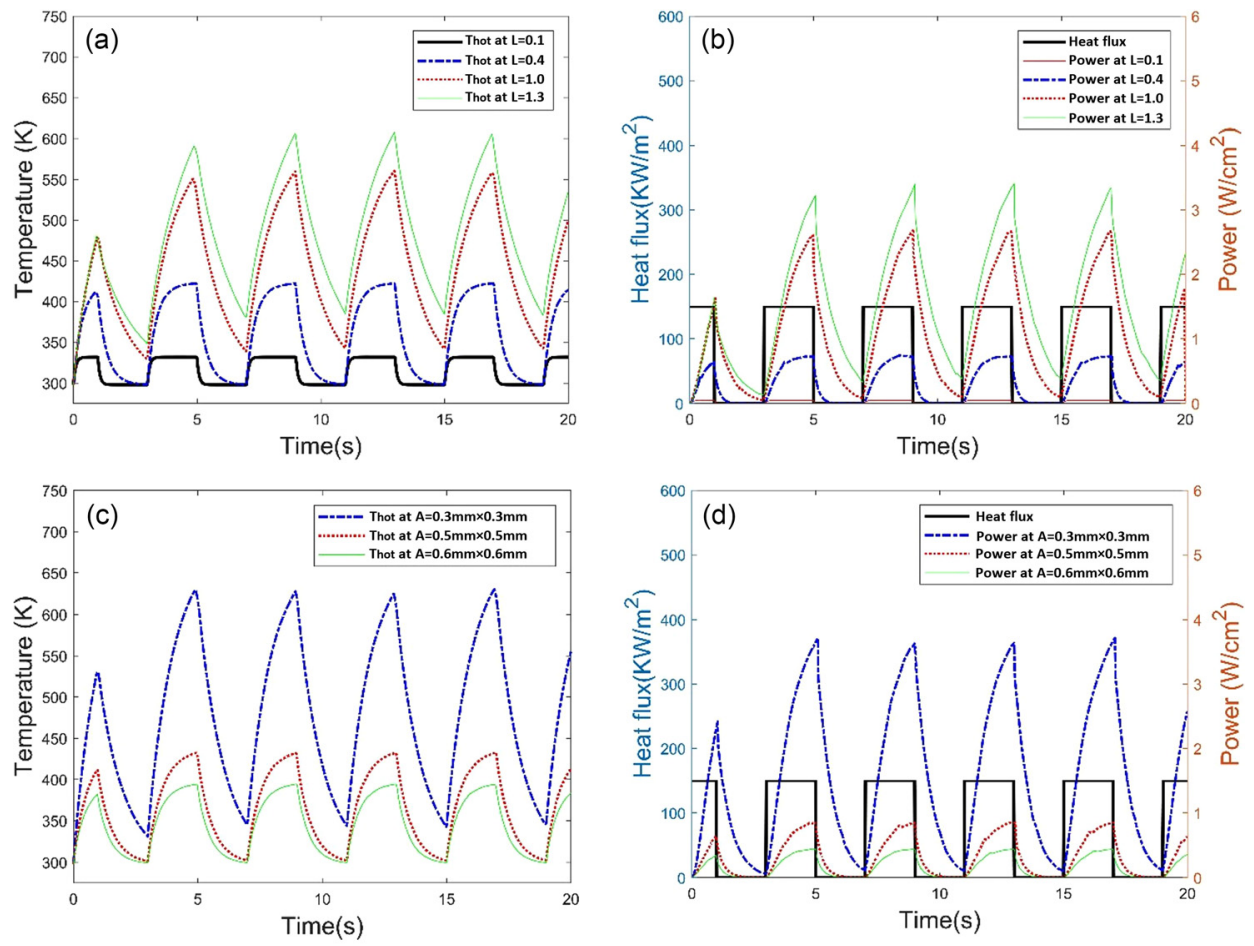

FIG. 7. Effect of TEG geometry on its performance with $\mathrm{f}=0.25 \mathrm{~Hz}$, (a) hot side temperature of TEG versus variation of the leg length, (b) applied heat flux and power generation for different leg lengths, (c) hot side temperature of TEG versus variation of the leg area, and (d) applied heat flux and power generation for different leg areas. 
indicate that the geometry of the TEG and properties of the heat flux (applied frequency in this study) have a significant effect on the electrical response of the device. Nevertheless, there are other critical parameters that need to be considered in the design of the optimal TEG module. As an example, although a shorter leg causes a higher temperature difference, optimal variation of figure of merit (ZT) of the materials needs to be taken into account in order to optimize the power generation since the ZT of most materials reduces after a specific temperature. Furthermore, the mechanical properties of the TEG module elements cause some restrictions, especially at high operating temperatures, and need to be carefully considered.

\section{CONCLUSIONS}

This study investigated the electrical response of TEGs under transient heat flux. Sensitivity of the TEG to the variation of critical design parameters, such as pulse shape, heat flux, and geometry of the TEG, is considered. The evaluation is carried out under a reference heat flux imposed on the hot surface of the TEG. The effect of frequency of the applied heat flux and geometry of the thermoelements on electrical response of the TEG is considered. The simulation results were in a good agreement with the experimental validation. The results show that the range of power oscillation reduces as the frequency of the imposed heat flux increases. Nevertheless, the maximum power generation enhances as the frequency reduces or the thermoelements have a longer length and a smaller cross-sectional area. The results, furthermore, show that oscillation of the voltage generation and output power is strongly dependent on the geometry of the TEG. The results of this study provide a guideline for geometry optimization of thermoelectric systems under transient heat sources.

\section{NOMENCLATURE}

A Area, $m^{2}$

$C$ heat capacity, J.(kg.K $)^{-1}$

$E$ electric field, V.m ${ }^{-1}$

$J$ electric current flux, A.m ${ }^{-2}$

$k$ thermal conductivity, W. $(\mathrm{m} . \mathrm{K})^{-1}$

$L \quad$ length, $m$

$P^{\prime} \quad$ Peltier coefficient

$Q^{\prime}$ density of Joule heating energy, W.m $\mathrm{m}^{-3}$

$q^{\prime \prime}$ heat flux, W.m ${ }^{-2}$

$R$ electrical resistance, $\Omega$

$S \quad$ Seebeck coefficient, V.K $\mathrm{K}^{-1}$

$T$ temperature, $\mathrm{K}$

$t$ time, $\mathrm{s}$

$V$ voltage, $\mathrm{V}$

$\dot{W}$ maximum power generation, $\mathrm{W}$

\section{Greek letters}

$\rho \quad$ density, kg.m ${ }^{-3}$

$\rho_{c}$ charge density, C.m ${ }^{-3}$

$\rho_{e}$ electrical resistivity, $\Omega . \mathrm{m}$

$\sigma$ electrical conductivity, S.m

\section{Subscripts}

n n-type

p p-type

${ }^{1}$ Z. Wang, V. Leonov, P. Fiorini, and C. Van Hoof, "Realization of a wearable miniaturized thermoelectric generator for human body applications," Sens. Actuators, A 156, 95-102 (2009). 
${ }^{2}$ S. Qing, A. Rezania, L. A. Rosendahl, A. A. Enkeshafi, and X. Gou, "Characteristics and parametric analysis of a novel flexible ink-based thermoelectric generator for human body sensor," Energy Convers. Manage. 156, 655-665 (2018).

${ }^{3}$ R. Amatya and R. Ram, "Solar thermoelectric generator for micropower applications," J. Electron. Mater. 39, 1735-1740 (2010).

${ }^{4}$ A. Elefsiniotis, N. Kokorakis, T. Becker, and U. Schmid, "A thermoelectric-based energy harvesting module with extended operational temperature range for powering autonomous wireless sensor nodes in aircraft," Sens. Actuators, A 206, 159-164 (2014)

${ }^{5}$ A. M. Goudarzi, P. Mazandarani, R. Panahi, H. Behsaz, A. Rezania, and L. A. Rosendahl, "Integration of thermoelectric generators and wood stove to produce heat, hot water, and electrical power," J. Electron. Mater. 42, 2127-2133 (2013).

${ }^{6} \mathrm{~J}$. C. Bass, N. B. Elsner, and F. A. Leavitt, "Performance of the $1 \mathrm{kw}$ thermoelectric generator for diesel engines," AIP Conf. Proc. 316, 295-298 (1994).

${ }^{7}$ F. Meng, L. Chen, Y. Feng, and B. Xiong, "Thermoelectric generator for industrial gas phase waste heat recovery," Energy 135, 83-90 (2017).

${ }^{8}$ H. Zhang, W. Kong, F. Dong, H. Xu, B. Chen, and M. Ni, "Application of cascading thermoelectric generator and cooler for waste heat recovery from solid oxide fuel cells," Energy Convers. Manage. 148, 1382-1390 (2017).

${ }^{9}$ Z.-G. Shen, S.-Y. Wu, and L. Xiao, "Assessment of the performance of annular thermoelectric couples under constant heat flux condition," Energy Convers. Manage. 150, 704-713 (2017).

${ }^{10}$ G. Kumar, S. Shashank, and K. P. Sudhir, "Calculation of efficiency and power output by considering different realistic prospects for recovering heat from automobile using thermoelectric generator," J. Renewable Sustainable Energy 9, 064703 (2017).

${ }^{11}$ X.-D. Jia, Y.-J. Wang, and Y.-W. Gao, "Numerical simulation of thermoelectric performance of linear-shaped thermoelectric generators under transient heat supply," Energy 130, 276-285 (2017).

${ }^{12}$ W.-H. Chen, P.-H. Wu, and Y.-L. Lin, "Performance optimization of thermoelectric generators designed by multiobjective genetic algorithm," Appl. Energy 209, 211-223 (2018).

${ }^{13}$ S. Mahmoudinezhad, A. Rezania, and L. A. Rosendahl, "Behavior of hybrid concentrated photovoltaic-thermoelectric generator under variable solar radiation," Energy Convers. Manage. 164, 443-452 (2018).

${ }^{14}$ S. A. Atouei, A. A. Ranjbar, and A. Rezania, "Experimental investigation of two-stage thermoelectric generator system integrated with phase change materials," Appl. Energy 208, 332-343 (2017).

${ }^{15}$ Y. Yan and J. A. Malen, "Periodic heating amplifies the efficiency of thermoelectric energy conversion," Energy Environ. Sci. 6, 1267-1273 (2013).

${ }^{16} \mathrm{O}$. Yamashita, H. Odahara, and K. Satou, "Energy conversion efficiency of a thermoelectric generator under the periodically alternating temperature gradients," J. Appl. Phys. 101, 023704 (2007).

${ }^{17}$ A. Rezania and L. A. Rosendahl, "Evaluating thermoelectric power generation device performance using a rectangular microchannel heat sink," J. Electron. Mater. 40, 481-488 (2011).

${ }^{18}$ A. Rezania and L. A. Rosendahl, "New configurations of micro plate-fin heat sink to reduce coolant pumping power," J. Electron. Mater. 41, 1298-1304 (2012).

${ }^{19} \mathrm{G}$. Min and D. Rowe, "Optimisation of thermoelectric module geometry for "waste heat' electric power generation," J. Power Sources 38, 253-259 (1992).

${ }^{20}$ A. Z. Sahin and B. S. Yilbas, "The thermoelement as thermoelectric power generator: Effect of leg geometry on the efficiency and power generation," Energy Convers. Manage. 65, 26-32 (2013).

${ }^{21}$ A. Rezania, L. Rosendahl, and H. Yin, "Parametric optimization of thermoelectric elements footprint for maximum power generation," J. Power Sources 255, 151-156 (2014).

${ }^{22} \mathrm{~L}$. Chen and J. Lee, "Effect of pulsed heat power on the thermal and electrical performances of a thermoelectric generator," Appl. Energy 150, 138-149 (2015).

${ }^{23}$ A. Rezania and L. A. Rosendahl, "A comparison of micro-structured flat-plate and cross-cut heat sinks for thermoelectric generation application,” Energy Convers. Manage. 101, 730-737 (2015).

${ }^{24} \mathrm{~T}$. Chai and R. R. Draxler, "Root mean square error (RMSE) or mean absolute error (MAE)?-arguments against avoiding RMSE in the literature," Geosci. Model Dev. 7, 1247-1250 (2014).

${ }^{25} \mathrm{Z}$. Ma, X. Wang, and A. Yang, "Influence of temperature on characters of thermoelectric generators based on test bed," J. Nanomater. 2014, 5 (2014) 